\title{
Effect of Leaves on the Regulation of Internode Elongation in a Tropical Evergreen Tree, Shorea leprosula
}

\author{
Masaki J. KOBAYASHI ${ }^{*}$, Kevin Kit Siong NG ${ }^{2}$, Soon Leong LEE $^{2}$, \\ Norwati MUHAMMAD ${ }^{2}$ and Naoki TANI ${ }^{1,3 *}$ \\ ${ }^{1}$ Japan International Research Center for Agricultural Sciences, Tsukuba, Japan \\ ${ }^{2}$ Forestry Biotechnology Division, Forest Research Institute Malaysia, Selangor Darul Ehsan, \\ Malaysia \\ ${ }^{3}$ Faculty of Life and Environmental Sciences, University of Tsukuba, Tsukuba, Japan
}

\begin{abstract}
Understanding internode elongation in woody species is the basis of stable timber production. Although trees exhibit coordinated growth between leaves and internodes, it is unclear whether their coordinated growth occurs simply because both organs receive a common signal that triggers their growth or because the growth of one organ is regulated by the other. To test the latter possibility, we defined simple developmental stages and assessed the effect of leaves on the regulation of internode elongation at the phytomer level in the tropical tree Shorea leprosula, which is an important timber tree species in Southeast Asia. Our observation of phytomers showed that leaves and internodes grew coordinately in S. leprosula. A spatiotemporal analysis of internode elongation indicated that internode elongation occurred around young expanding leaves. Furthermore, the excision of expanding leaves significantly reduced internode elongation. These results suggest that expanding leaves positively regulate internode elongation. Therefore, the growth interactions between leaves and internodes must be considered in order to better understand the mechanism of internode elongation in S. leprosula. These results will also form the basis of future studies aimed at sustainable timber production using this species.
\end{abstract}

Discipline: Forestry

Additional key words: development, Dipterocarpaceae, evergreen tree, stem elongation, tropical timber tree

\section{Introduction}

In many plant species, stem or internode elongation has been extensively studied as an important developmental process that affects plant height (Sachs 1965, Cosgrove \& Sovonick-Dunford 1989, Ruonala et al. 2008, Dayan et al. 2012, Euring et al. 2014, Procko et al. 2014, Nito et al. 2015, Simon et al. 2018, Bellstaedt et al. 2019, Patil et al. 2019), which is a key trait in agriculture and forestry due to its impact on the yield and biomass of crops and timber (Eriksson et al. 2000, Dubouzet et al. 2013, Mathan et al. 2016). These studies have revealed that stem or internode elongation is regulated by complex mechanisms involving many factors, such as nutrients, plant hormones, environmental factors, and interorgan communication (Sachs 1965,
Cosgrove \& Sovonick-Dunford 1989, Ruonala et al. 2008, Dayan et al. 2012, Euring et al. 2014, Procko et al. 2014, Nito et al. 2015, Simon et al. 2018, Bellstaedt et al. 2019, Patil et al. 2019).

During shoot development, trees exhibit coordinated growth between leaves and internodes (Meng et al. 2015). However, it is not clear how this coordinated growth occurs. If common regulators (e.g., environmental factors and plant hormones) regulate the growth of leaves and internodes (Vitasse et al., 2009, Dayan et al. 2012), their simultaneous growth will be observed when both receive signals from the regulators at the same time. In this case, because such simultaneous growth can occur without their interactions, the mechanism of internode elongation can be understood by studying only internodes. However, if the coordinated

*Co-corresponding authors: masakijkobayashi@affrc.go.jp,ntani@affrc.go.jp

Received 22 July 2020; accepted 17 September 2020. 
growth occurs because leaf growth regulates internode growth or vice versa, meaning that interorgan regulation exists between leaves and internodes regarding the control of their growth, the development of both internodes and leaves must be studied simultaneously to fully understand the mechanism of internode elongation. In plants, the participation of organ interactions in the regulation of organ growth has been reported in multiple organs and species (Kozuka et al. 2010, Dayan et al. 2012, Casal 2013, Procko et al. 2014, Nito et al. 2015, Bellstaedt et al. 2019). Therefore, whether such interorgan regulatory mechanisms are involved in the shoot development in trees must be carefully examined in order to elucidate the mechanism of internode elongation.

For this purpose, we studied the effect of developing leaves on the regulation of internode elongation in a tropical timber tree species (Shorea leprosula) in the family Dipterocarpaceae. Dipterocarpaceae is a dominant family in the tropical rainforests of Southeast Asia and one of the most important timber sources in this region (Ashton 1988, Appanah \& Turnbull 1998, Ghazoul 2016). Among the more than 500 species in this family, S. leprosula is a commercially well-known and widely distributed timber species (Ashton 1988, Appanah \& Turnbull 1998, Ghazoul 2016). Using this species, we 1) defined the simple developmental stages to explain the shoot development of $S$. leprosula, 2) examined the relationship between leaf development and internode elongation during shoot development using the introduced developmental stages, 3) assessed the spatiotemporal pattern of internode elongation around a developing leaf, and 4) performed leaf excision during internode elongation to test the effects of developing leaves on this trait. By integrating the results of these experiments, we discuss the possible mechanisms underlying the coordinated growth observed between leaves and internodes in S. leprosula.

\section{Materials and methods}

\section{Plant materials and growth conditions}

In this study, we used S. leprosula seedlings of the same age ( $\sim 5$ years old) collected from the same mother tree grown at the Forest Research Institute Malaysia (FRIM; $3^{\circ} 14^{\prime} \mathrm{N}, 101^{\circ} 38^{\prime} \mathrm{E}$ ). The seedlings were placed in plastic pots ( $23 \mathrm{~cm}$ in diameter, $28 \mathrm{~cm}$ in height) in the nursery at FRIM, exposed to direct sunlight, and watered twice a day (at 0700 and 1700).

\section{Observation of $\boldsymbol{S}$. leprosula phytomers}

A shoot consists of a sequence of structural units known as phytomers, each of which possesses a node, a leaf at the node, and an internode subtending the leaf (Howell 1998) (Supplementary Fig. S1 (A)). We therefore studied the relationship between leaf development and internode elongation at the phytomer level in S. leprosula using lateral shoots. For this purpose, we monitored whether leaves were folded or unfolded on a weekly basis, and measured the length of leaves and internodes using a digital caliper (Mitutoyo, Tokyo, Japan). Although the timing of leaf production, which corresponds to the timing of phytomer growth, has been studied in dipterocarps including S. leprosula (Medway 1972, Ng 1981), predicting the onset and end of phytomer growth under natural environments remains difficult. In order to overcome the unpredictability of phytomer growth and obtain measurement data during the onset and termination of phytomer growth, we performed observations over two periods (June 6 to July 4 and September 12 to October 3, 2017). During the first observation period, we obtained 133 measurements from 43 different phytomers using 12 lateral shoots of seven individuals. During the second observation period, we obtained 97 measurements from 34 different phytomers using seven lateral shoots of six individuals. A total of 230 measurements were performed during both observation periods, with an average of four phytomers being measured in each shoot. Based on these measurements, four developmental stages of phytomers (S1, S2, S3, and S4) were introduced. The Results section describes the developmental stages in detail.

\section{Analysis of the spatiotemporal internode elongation around $\mathbf{S 2}$ leaves in $\mathbf{S}$. leprosula}

To analyze the spatiotemporal internode elongation around S2 leaves in S. leprosula, we focused on the shoots that consisted of two successive phytomers: the distal and proximal phytomers at the S1 and S2 stages. We introduced ink spots on the successive internodes at intervals of about $2 \mathrm{~mm}$ and acquired images of the internodes at 4-day intervals over 12 days. The distance between successive ink spots was measured in the pictures obtained using ImageJ software (version 1.50i; https://imagej.nih.gov/ij/). Their relative growth was then calculated based on a comparison with their initial length (i.e., relative growth $=$ observed length / initial length). To display representative images of internode elongation, images were trimmed using Adobe Photoshop Elements 15 (Adobe Systems Inc., San Jose, CA, USA).

\section{Excision of leaves to test their effects on internode elongation}

To test the effects of leaves on internode elongation 
at the S2 stage in S. leprosula, we examined the relative growth of internodes under (i) no treatment and (ii) leafexcision conditions over one week. To calculate the relative growth, we used a digital caliper to measure the length of the internodes before and after each experiment. The leaf-excision treatment was conducted by removing an S2 leaf at the distal end of each examined internode prior to each experiment. Two experiments were conducted: one from February 2 to 9, 2018 and the other from December 6 to 13, 2019. We conducted experiments during these periods as multiple phytomers at the S2 stage could easily be observed. We used 17 and 22 internodes for each condition in the first and second experiments, respectively.

\section{Statistical analysis}

To test the statistical significance of organ elongation at the S1, S2, and S3 stages, $t$-tests were performed based on comparisons with the S4 stage (as a control) by considering multiple comparisons using $\mathrm{R}$ 3.5.0 (R Core Team). $P$-values for multiple comparisons were adjusted using Bonferroni correction. For the leafexcision experiment, the statistical significance of growth differences between the control and treatment conditions was also evaluated via a $t$-test using R. In both cases, significance was set at $P<0.05$.

\section{Results}

\section{Introduction of four developmental stages of S. leprosula phytomers}

To understand the relationship between leaf development and internode elongation during shoot development, we observed the phytomers of S. leprosula weekly. Based on this observation, we found that leaves unfolded as they developed (Supplementary Fig. S1 (A) and (B)). An examination of the elongation rate of leaves revealed clear differences in distribution patterns between an elongation rate of less than $5 \mathrm{~mm}$ in one week and others, regardless of whether leaves were folded or unfolded (Supplementary Fig. S2). Therefore, using these morphological characteristics (i.e., folded, unfolded) and the elongation rate of the leaves, we classified S. leprosula phytomers into four developmental stages: (i) S1, leaves were folded and elongated less than $5 \mathrm{~mm}$ in one week; (ii) S2, leaves were folded and elongated 5 $\mathrm{mm}$ or more in one week; (iii) S3, leaves were unfolded and elongated $5 \mathrm{~mm}$ or more in one week; and (iv) S4, leaves were unfolded and elongated less than $5 \mathrm{~mm}$ in one week. Among the 230 phytomers observed, we found $69,53,38$, and 70 phytomers in the S1, S2, S3, and S4 stages, respectively. As observed in Supplementary
Figure S1 (A) and (B), the development of phytomers basically progressed in order of S1, S2, S3, and S4, and phytomers at earlier developmental stages tended to be found near the tips of shoots. The average leaf length at the S1, S2, S3, and S4 stages was 9.2, 19.5, 67.1, and $103.9 \mathrm{~mm}$, respectively. The average internode length at the S1, S2, S3, and S4 stages was 5.9, 9.1, 16.8, and $16.0 \mathrm{~mm}$, respectively.

\section{Developmental relationships between leaves and internodes on $S$. leprosula phytomers}

Using the developmental stages defined herein, we checked the elongation rates of leaves and internodes to test whether they grow coordinately. In this analysis, we used leaves and internodes at $\mathrm{S} 4$ as controls for comparisons of elongation rates at different developmental stages, as both exhibited modest elongation (Fig. 1 (A) and (B)). During shoot development, leaves at S2 and S3 were significantly elongated, while leaves at $\mathrm{S} 1$ were not, compared with leaves at S4 (Fig. 1 (A)). Similarly, internodes at S2 were significantly elongated (Fig. 1 (B)), indicating that clear growth of both leaves and internodes had occurred at the S2 stage. Conversely, internodes at S3 were not significantly elongated (Fig. 1 (B)), although the leaves at S3 were significantly elongated (Fig. 1 (A)). In contrast, we observed significant internode elongation at the S1 stage. However, the elongation rate of internodes detected at S1 was much lower than that observed at S2 (Fig. 1 (B)). To understand the observed elongation of S1 internodes in greater detail, we tested whether the elongation of S1 internodes was affected by the developmental stages of their adjacent phytomers. When $\mathrm{S} 2$ and S3 phytomers were observed at the proximal sides of S1 internodes, the S1 internodes showed significant elongation (S1S2 and S1S3 in Fig. 1 (C)) compared with those next to S4 phytomers (S1S4 in Fig. 1 (C)), while such elongation was not observed when S1 phytomers were found at the proximal sides of S1 internodes (S1S1 in Fig. 1 (C)). This indicates that internode elongation at $\mathrm{S} 1$ varies in the developmental stages of their adjacent phytomers, suggesting the presence of context-dependent S1 internode elongation.

\section{Spatiotemporal internode elongation in S. leprosula}

To analyze the S2 internode elongation and contextdependent S1 internode elongation, we performed a spatiotemporal analysis of internode elongation by focusing on shoots at which the S1 and S2 phytomers were located at the distal and proximal sides, respectively (phytomers A and B in Fig. 2 (A), 

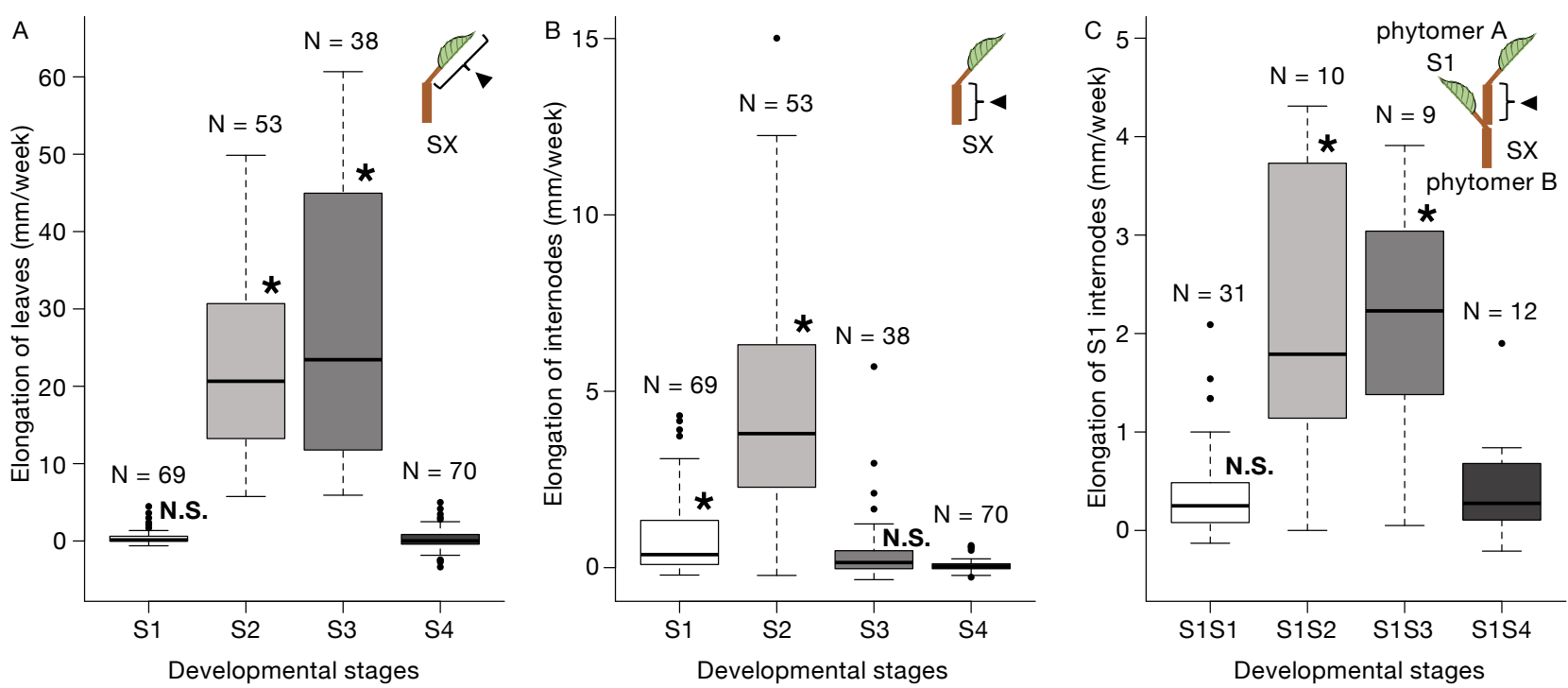

Fig. 1. Elongation of leaves and internodes at different developmental stages in Shorea leprosula

(A) Elongation of leaves in one week at the four developmental stages. The $x$-axis indicates the developmental stages of the leaves studied. (B) Elongation of internodes in one week at the four developmental stages. The $x$-axis indicates the developmental stages of the studied internodes. (C) Elongation of S1 internodes (phytomers A) in one week considering the developmental stage of adjacent phytomers (phytomers B) at the proximal side. The adjacent phytomers at the distal and proximal sides were defined as phytomers $\mathrm{A}$ and $\mathrm{B}$, respectively. The $x$-axis indicates the developmental stages of the successive phytomers. When the developmental stages of phytomers A and B were S1 and S2, respectively, the developmental stages of these successive phytomers were indicated as S1S2. Among the 69 S1 internodes observed in Figure 1 (B), seven internodes were excluded from this analysis because the developmental stages of their adjacent phytomers (phytomers B) were not identified. $\mathrm{N}$ indicates the number of samples observed. In each figure, asterisks indicate statistically significant differences compared with S4 or S1S4 ( $P$-value cutoff by $t$-test: 0.05 after Bonferroni correction). N.S. means not significant. In each figure, the region used for measurement is indicated by an arrowhead in the schematic image included in the upper-right corner. "SX" in the schematic image indicates any of the S1-S4 developmental stages.

respectively). This analysis revealed that the internode of phytomer B elongated more at its distal side, whereas the internode of phytomer A elongated more at its proximal side (Fig. 2). Therefore, we found that internode elongation tended to occur at a higher rate in regions near the developing leaves of phytomer B and gradually decreased toward both ends (Fig. 2 and Supplementary Fig. S3).

\section{Assessment of the effects of leaves on the elongation of $\mathbf{S 2}$ internodes in $\mathbf{S}$. leprosula}

Finally, we conducted leaf-excision experiments to test the effects of the S2 leaves on internode elongation, by removing S2 leaves from the distal ends of S2 internodes. The results showed that removal of the S2 leaves led to a significant reduction in the elongation of internodes compared with the control S2 internodes, regardless of the different periods when the experiments were started (Fig. 3 (A) and (B)), thereby suggesting that the S2 leaves have positive effects on internode elongation. However, it should be noted that the relative growth of internodes, which was expressed as the ratio of internode length before and after each experiment, was slightly higher than 1 under the leaf-excision condition (Fig. 3 (A) and (B)), suggesting that internode elongation cannot be completely suppressed by the excision of S2 leaves, and that the effects of adjacent phytomers or other factors might affect the slight growth of internodes under the leaf-excision condition.

\section{Discussion}

S. leprosula is an important timber species in Southeast Asia (Ashton 1988, Appanah \& Turnbull 1998, Ghazoul 2016). In this species, the timing of leaf production, which corresponds to the timing of internode elongation, has been studied for several decades (Medway 1972, Ng 1981). However, it remains unclear how internodes develop in this species. In order to understand the regulation of internode elongation by leaves, we first defined four developmental stages of the S. leprosula phytomers (i.e., S1, S2, S3, S4) based on the 


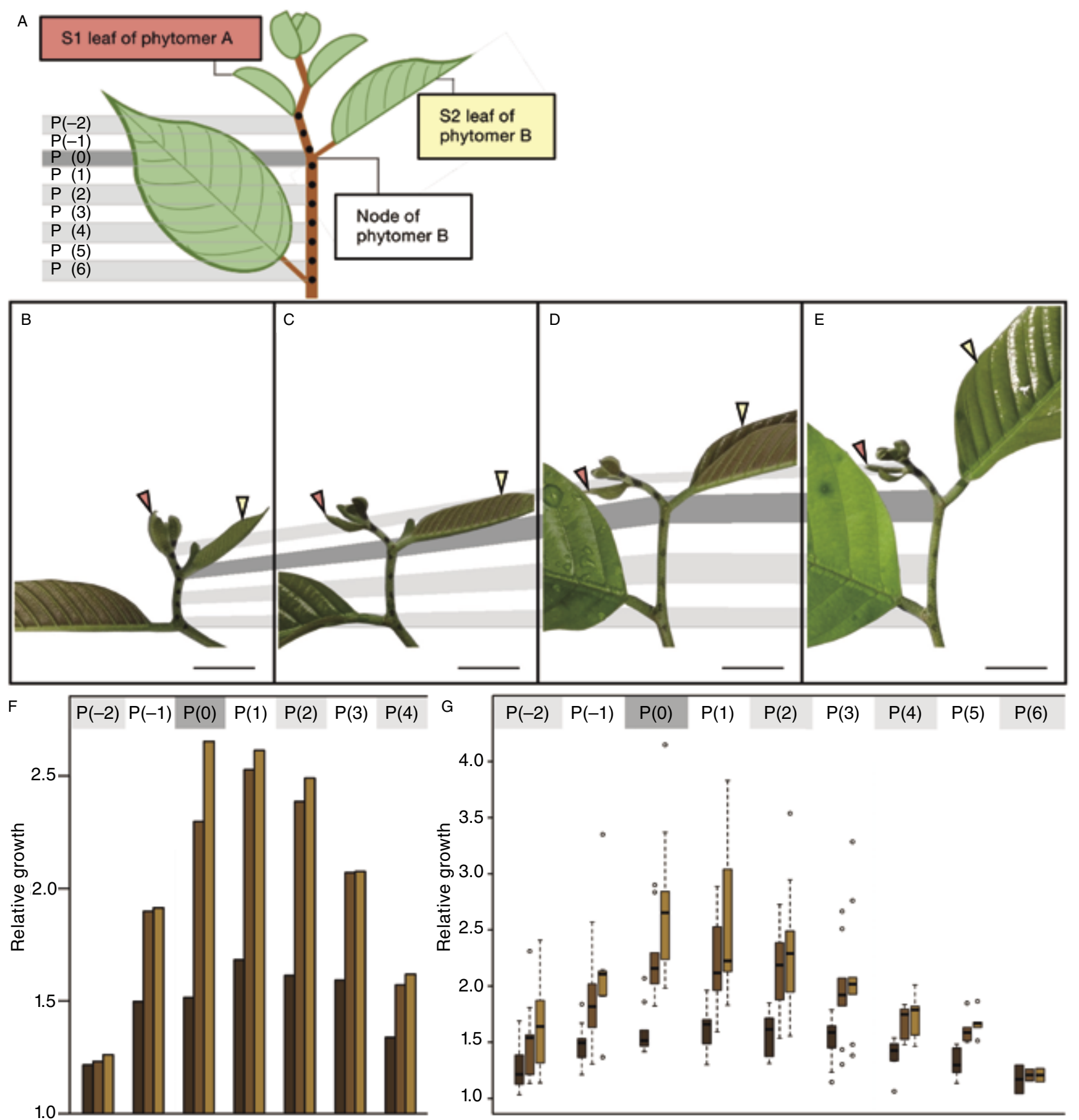

Fig. 2. Spatiotemporal analysis of internode elongation around a developing leaf in Shorea leprosula

(A) Schematic representation of an observed shoot. When the observation began, the analyzed region consisted of two adjacent phytomers, with the distal and proximal ones being at the S1 (phytomer A: red) and S2 (phytomer B: yellow) stages, respectively. The ink spots on the internode were introduced at about 2-mm intervals. We defined the position at which the node of phytomer B was included as $\mathrm{P}(0)$. The positions at the distal (phytomer A) and proximal (phytomer B) sides of $\mathrm{P}(0)$ are indicated by negative and positive values of $X$ in $P(X)$, respectively. (B-E) Internode elongation around a developing leaf of phytomer B recorded at 4-day intervals. The red and yellow arrowheads indicate the leaves of phytomers A and B, respectively. Scale bars: $1 \mathrm{~cm}$. (F) Spatiotemporal pattern of internode elongation in the representative lateral shoot shown in (C-E). In each $\mathrm{P}(\mathrm{X})$ position, the three bar plots (from left to right) show the relative growth at $\mathrm{P}(\mathrm{X})$ of the lateral shoot at 4, 8, and 12 days after initial observation. (G) Box plots representing the trend of the spatiotemporal internode elongation patterns obtained from the nine shoots shown in (F) and Supplementary Fig. S3 (B), (D), (F), (H), (J), $(\mathrm{L}),(\mathrm{N})$, and $(\mathrm{P})$. At each $\mathrm{P}(\mathrm{X})$ position, the three box plots (from left to right) show the relative growth at $\mathrm{P}(\mathrm{X})$ of nine shoots at 4,8 , and 12 days after initial observation. The position of $\mathrm{P}(0)$ is shown in dark gray on the images. The positions with even numbers of $\mathrm{X}$ are highlighted in light gray on the images. 

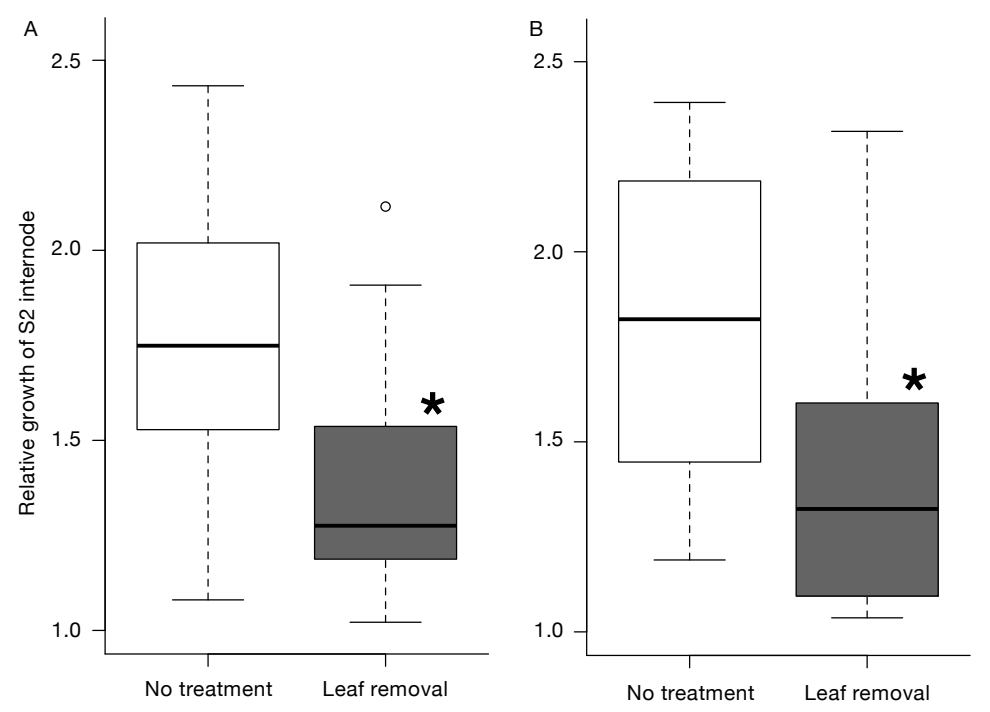

Fig. 3. Effect of leaves on the elongation of S2 internodes

Results of the leaf-excision experiments conducted from February 2 to 9, 2018 (A) and from December 6 to 13, 2019 (B). The boxes in light gray and white represent the relative growth of S2 internodes after one week of observation under no treatment and leaf-excision treatment, respectively. In each figure, asterisks indicate statistically significant differences compared with the no-treatment condition ( $P$-value cutoff by $t$-test: 0.05$)$.

morphology and elongation rate of leaves on the phytomers, and found that these developmental stages captured well the developmental progression of the $S$. leprosula phytomers (Supplementary Fig. S1). At the S1 stage, neither leaves nor internodes showed significant elongation when adjacent phytomers at the proximal side were at the S1 or S4 stage (Fig. 1 (B) and (C)). Furthermore, S1 phytomers tended to remain at the same stage for several weeks (Supplementary Fig. S1 (C)). These results suggest that $\mathrm{S} 1$ is the growth-arrest stage. Conversely, both S2 and S3 phytomers showed significant leaf elongation (Fig. 1 (A)), although internode elongation was mainly observed at the S2 stage (Fig. 1 (B)). These results indicate that the vigorous growth of both leaves and internodes starts at S2, but that internode elongation ends earlier than does leaf expansion. At the S4 stage, neither leaves nor internodes showed clear elongation (Fig. 1 (A) and (B)), and phytomers at the S4 stage did not change their developmental stage (Supplementary Fig. S1), indicating that $\mathrm{S} 4$ corresponds to the mature stage.

Our analysis revealed that both leaves and internodes were significantly elongated at the S2 stage (Fig. 1 (A) and (B)). However, the removal of the developing S2 leaves led to a significant reduction in the elongation of internodes (Fig. 3). These results suggest that S2 leaves have positive effects on internode elongation, and that the coordinated growth observed between leaves and internodes at the S2 phytomers can be partly explained by the leaf-dependent growth of internodes. Our spatiotemporal analysis of internode elongation showed that internodes located around the S2 leaves tended to grow (Fig. 2, Supplementary Fig. S3). A possible mechanism to explain the observed leafdependent internode growth is that developing S2 leaves produce substances that promote the growth of internodes. The presence of such growth-promoting substances and the gradual decrease of their concentrations toward both ends of internodes from the S2 leaves would explain the observed spatiotemporal pattern of internode elongation. A recent study using a herbaceous plant, tobacco (Nicotiana tabacum), showed that leaf-derived gibberellin is required for stem elongation during the vegetative stage (Dayan et al. 2012). As this finding is similar to our observations of $S$. leprosula, plant hormones such as gibberellin may also act as growth-promoting substances provided in the leaves to regulate internode elongation in S. leprosula. Another possibility is that the young developing S2 leaves of $S$. leprosula indirectly regulate internode elongation by acting as a sink in promoting the translocation of assimilates. If the developing S2 leaves cause a gradient of assimilates from the basal part of the shoot to the developing S2 leaves and an inverse- 
direction gradient of other factor(s) exists, the highest overlap of these gradients should be observed around the developing S2 leaves, so as to decrease gradually according to distance from the leaves. For example, the concentration of auxin is known to gradually decrease from the tip of shoots to their basal parts (Muday \& DeLong 2001). Therefore, if a combination of these factors (i.e., assimilates, auxin) promotes internode elongation, the observed pattern of internode elongation can be explained by the overlapping of their inverse gradients. As both possible mechanisms are not mutually exclusive, it is also plausible to expect that the developing leaves act as both direct and indirect regulators of internode elongation in S. leprosula. An analysis of the internal states of both leaves and internodes (e.g., nutrient, plant hormone, geneexpression levels) will elucidate details of the mechanism underlying the leaf-dependent internode elongation in $S$. leprosula.

Although further studies are needed to understand the mechanism underlying this phenomenon, our results showed the occurrence of spatiotemporal internode elongation around developing leaves (Fig. 2), and clearly demonstrated that the removal of developing leaves significantly reduced internode elongation in $S$. leprosula (Fig. 3). These results suggest the importance of growth interactions between leaves and internodes in the regulation of internode elongation in S. leprosula. We believe that our results will promote a better understanding of the mechanism of internode elongation and form the basis of future studies of sustainable timber production from $S$. leprosula.

\section{Supplementary data}

Fig. S1. Shoot development in Shorea leprosula

Fig. S2. Elongation of folded and unfolded leaves during a one-week period in Shorea leprosula

Fig. S3. Spatiotemporal analysis of internode elongation around a developing leaf in Shorea leprosula

\section{Acknowledgements}

We wish to thank Drs. I. Harun, S. Musa, I. Parlan, Y. Yamamoto, G. Takao, and H. Oka for project management; and Dr. C. H. Ng and Mr. G. Jaafar, Y. Marhani, R. Ponyoh, and Y. Baya for field assistance. The study was supported by a FRIM-JIRCAS joint project entitled "Development of value-adding techniques for dipterocarp tree products by utilizing the genetic resources" (C-4-2).

\section{References}

Appanah, S. \& Turnbull, J. M. (1998) A Review of Dipterocarps: Taxonomy, Ecology and Silviculture. Center for International Forestry Research, Bogor, Indonesia.

Ashton, P. S. (1988) Dipterocarp biology as a window to the understanding of tropical forest structure. Annual Review of Ecology and Systematics, 19, 347-370.

Bellstaedt, J. et al. (2019) A mobile auxin signal connects temperature sensing in cotyledons with growth responses in hypocotyls. Plant Physiology, 180, 757-766.

Casal, J. J. (2013) Photoreceptor signaling networks in plant responses to shade. Annual Review of Plant Biology, 64, 403-427.

Cosgrove, D. J. \& Sovonick-Dunford, S. A. (1989) Mechanism of gibberellin-dependent stem elongation in peas. Plant Physiology, 89, 184-191.

Dayan, J. et al. (2012) Leaf-induced gibberellin signaling is essential for internode elongation, cambial activity, and fiber differentiation in tobacco stems. The Plant Cell, 24, 66-79.

Dubouzet, J. G. et al. (2013) Potential transgenic routes to increase tree biomass. Plant Science, 212, 72-101.

Eriksson, M. E. (2000) Increased gibberellin biosynthesis in transgenic trees promotes growth, biomass production and xylem fiber length. Nature Biotechnology, 18, 784-788.

Euring, D. et al. (2014) Nitrogen-driven stem elongation in poplar is linked with wood modification and gene clusters for stress, photosynthesis and cell wall formation. $B M C$ Plant Biology, 14, 391.

Ghazoul, J. (2016) Dipterocarp Biology, Ecology, and Conservation. Oxford University Press, Oxford, UK.

Howell, S. H. (1998) Molecular Genetics of Plant Development. Cambridge University Press, Cambridge, MA.

Kozuka, T. et al. (2010) Involvement of auxin and brassinosteroid in the regulation of petiole elongation under the shade. Plant Physiology, 153, 1608-1618.

Mathan, J. et al. (2016) Enhancing crop yield by optimizing plant developmental features. Development, 143, 3283-3294.

Medway, L. (1972) Phenology of a tropical rain forest in Malaya. Biological Journal of the Linnean Society, 4, 117-146.

Meng, F. et al. (2015) Growth synchrony between leaves and stems during twig development differs among plant functional types of subtropical rainforest woody species. Tree Physiology, 35, 621-631.

Muday, G. K. \& DeLong, A. (2001) Polar auxin transport: controlling where and how much. Trends in Plant Science, 6, 535-542.

Ng, F. S. P. (1981) Vegetative and reproductive phenology of dipterocarps. Malayan Forester, 44, 197-221.

Nito, K. et al. (2015) Spatial regulation of the gene expression response to shade in Arabidopsis seedlings. Plant and Cell Physiology, 56, 1306-1319.

Patil, V. et al. (2019) APETALA2 control of barley internode elongation. Development, 146, dev170373.

Procko, C. et al. (2014) Cotyledon-generated auxin is required for shade-induced hypocotyl growth in Brassica rapa. Plant Physiology, 165, 1285-1301.

Ruonala, R. et al. (2008) CENL1 expression in the Rib meristem affects stem elongation and the transition to 
dormancy in Populus. The Plant Cell, 20, 59-74.

Sachs, R. M. (1965) Stem elongation. Annual Review of Plant Physiology, 16, 73-96.

Simon, N. M. L. et al. (2018) The energy-signaling hub SnRK1 is important for sucrose-induced hypocotyl elongation.
Plant Physiology, 176, 1299-1310.

Vitasse, Y. et al. (2009) Leaf phenology sensitivity to temperature in European trees: do within-species populations exhibit similar responses? Agricultural and Forest Meteorology, 149, 735-744.

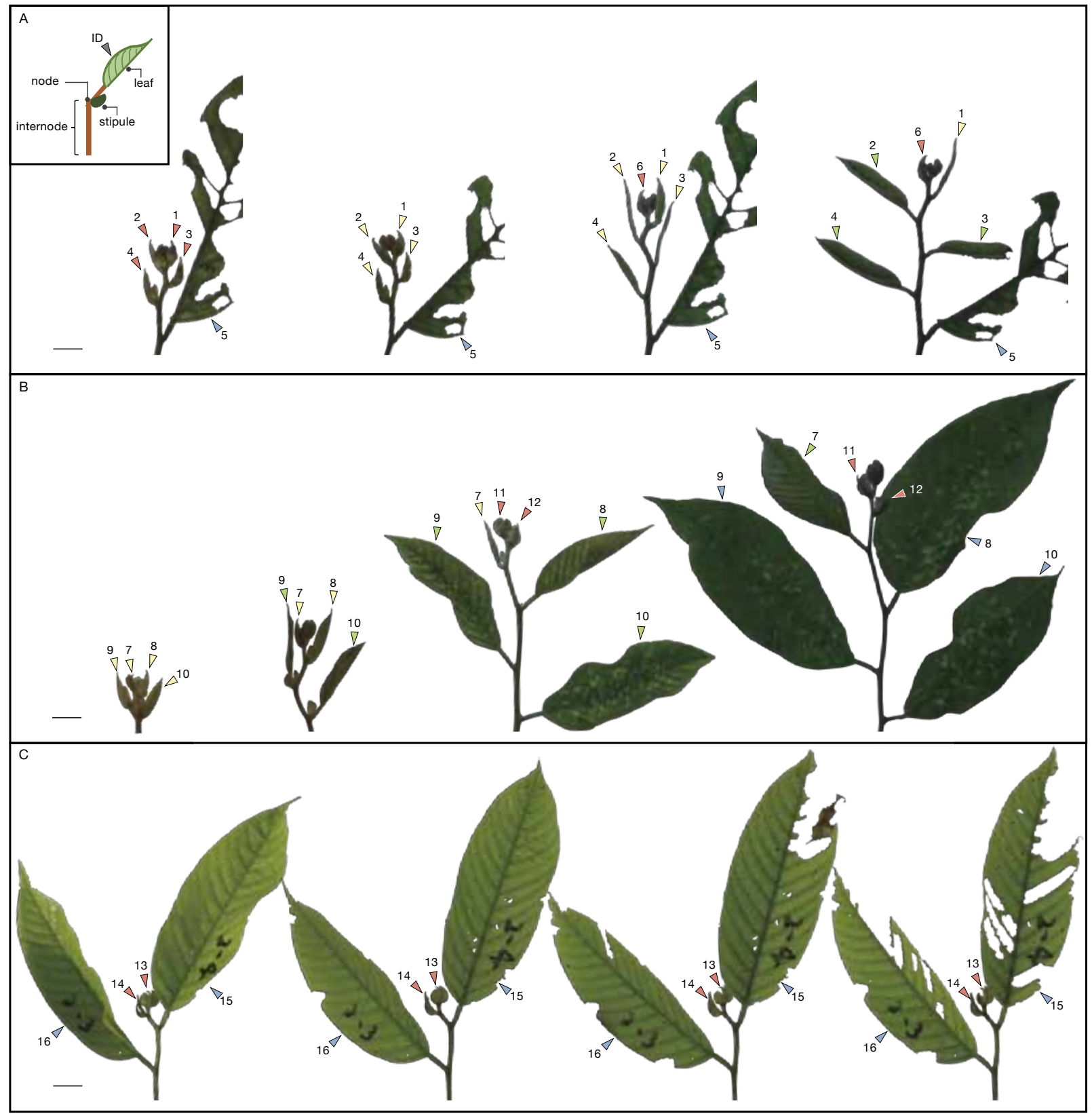

Fig. S1. Shoot development in Shorea leprosula

Shoots of S. leprosula during the earlier growth (A), later growth (B), and growth-arrest (C) phases over three weeks. In each figure, morphological changes in the observed shoot at weeks $0,1,2$, and 3 are shown from left to right. The schematic image presented in the upper-left corner of (A) represents an example of a phytomer in S. leprosula. Each phytomer is indicated by arrowheads with ID numbers. The same phytomers in different weeks are represented by the same ID numbers. The colors of arrowheads indicate the developmental stages of phytomers: red: S1; yellow: S2; green: S3; and blue: S4. For example, the developmental stages of phytomer 2 in (A) were S1, S2, S2, and S3 at weeks $0,1,2$, and 3, respectively, indicating a gradual developmental progression. Conversely, the developmental stages of phytomers 13 and 15 in (C) did not change from S1 and S4, respectively, during the three-week observation period. The developmental stages of phytomers from the tip of the shoot in (B) at week 3 were S1 (phytomer 11), S1 (phytomer 12), S2 (phytomer 7), S3 (phytomer 8), S3 (phytomer 9), and S3 (phytomer 10), indicating that phytomers at earlier developmental stages tended to be found near the tip of the shoot. Scale bars: $1 \mathrm{~cm}$. 

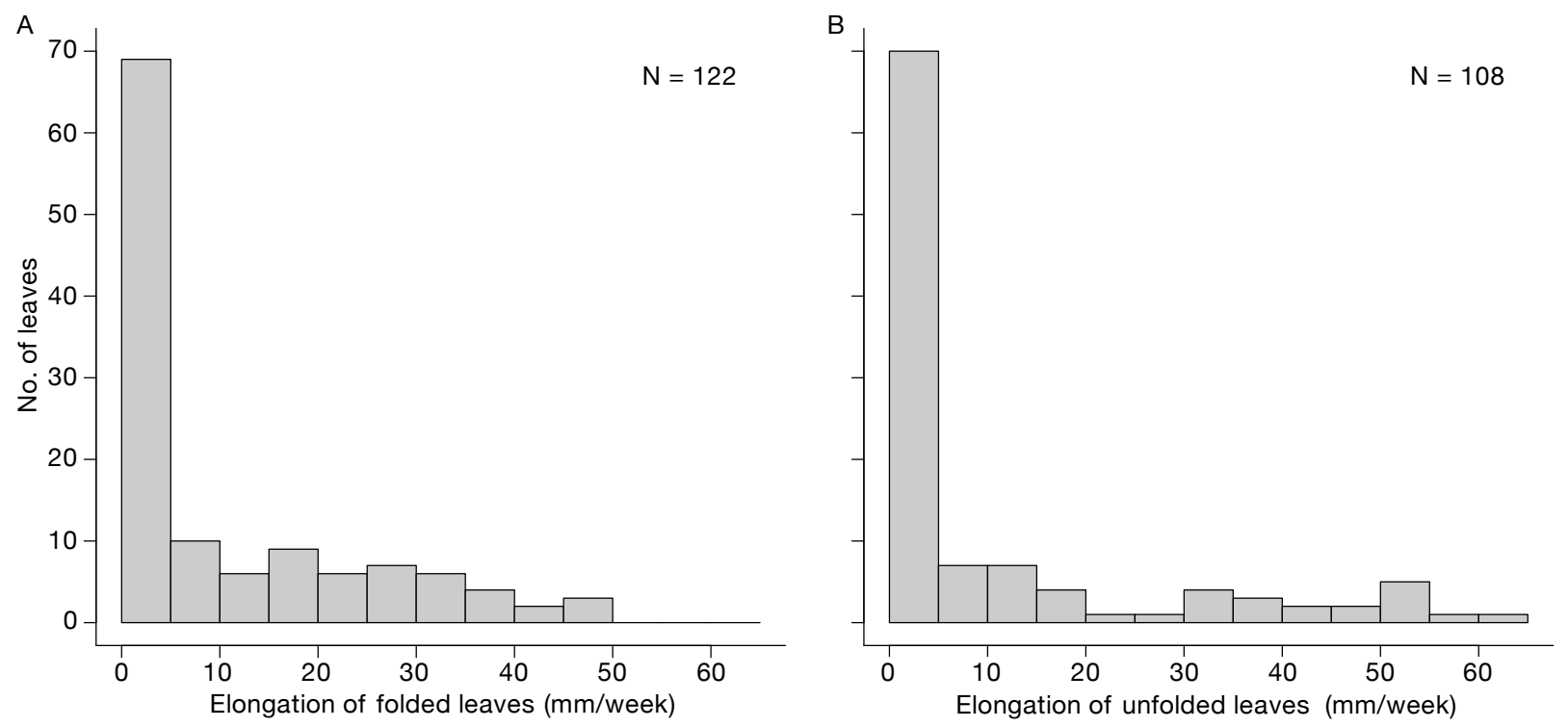

Fig. S2. Elongation of folded and unfolded leaves during a one-week period in Shorea leprosula Distribution patterns of the elongation rate of folded (A) and unfolded (B) leaves. N indicates the number of leaves observed. 

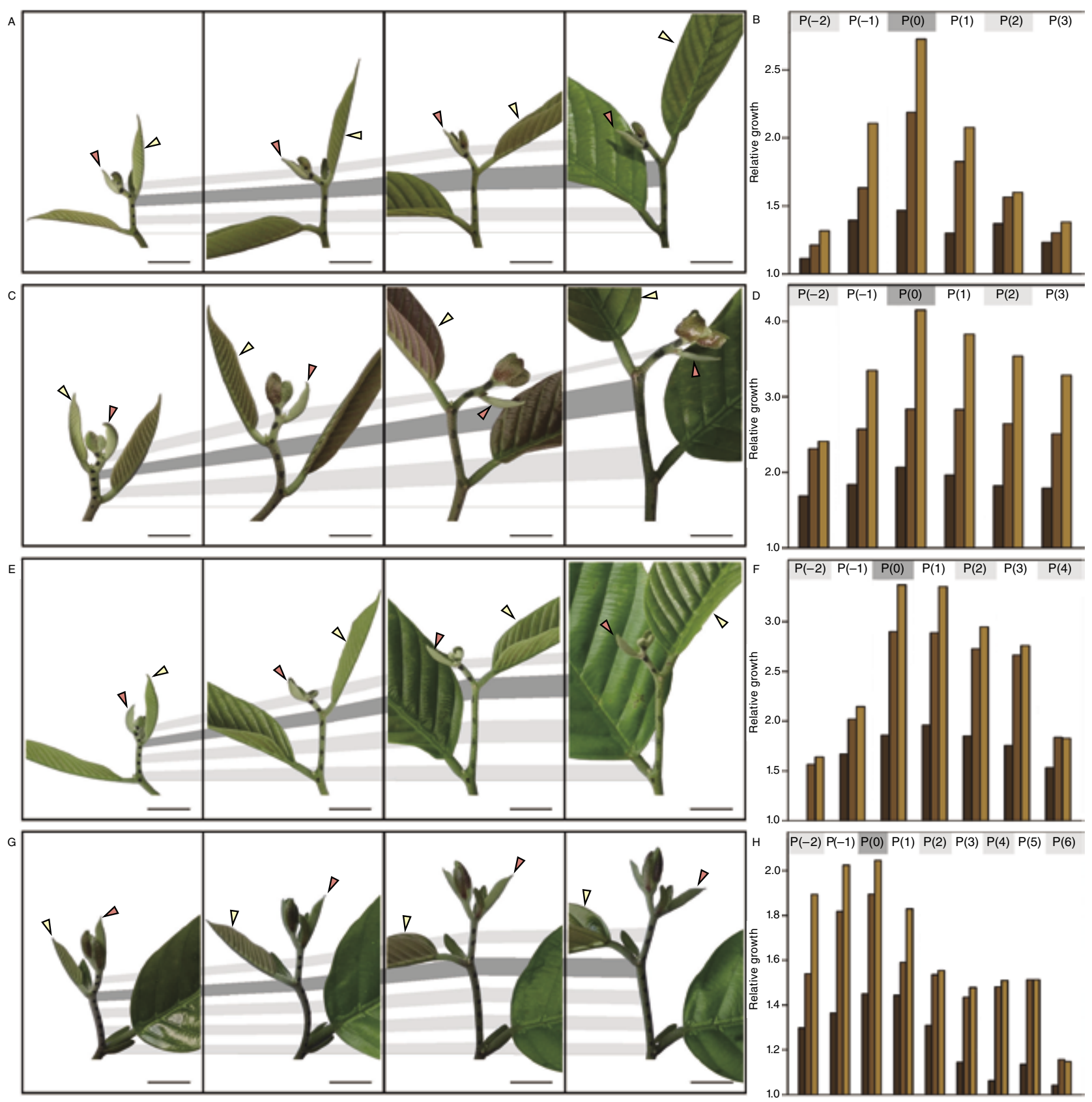

(Continued on next page ) 
( Continued )
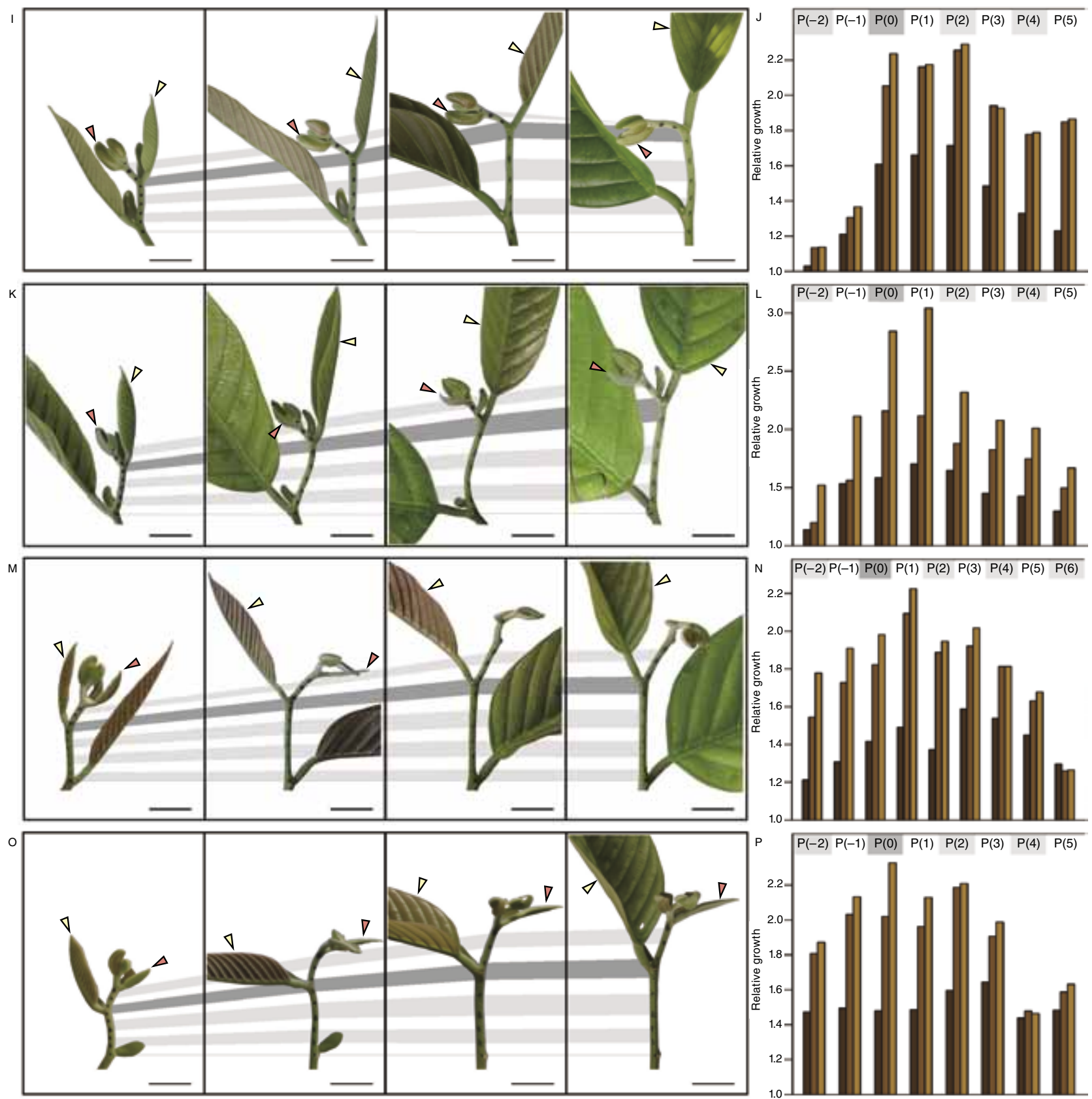

Fig. S3. Spatiotemporal analysis of internode elongation around a developing leaf in Shorea leprosula

Images of internode elongation around a developing leaf at 4-day intervals (A, C, E, G, I, K, M, and O). When the observation began, the analyzed region consisted of two adjacent phytomers, with the distal and proximal ones being at the S1 (phytomer A) and S2 (phytomer B) stages, respectively. The red and yellow arrowheads indicate adjacent phytomers A and B, respectively. The ink spots on the internode were introduced at about 2-mm intervals. We defined the position at which the node of phytomer B was included as $\mathrm{P}(0)$. The positions at the distal (phytomer $\mathrm{A}$ ) and proximal (phytomer $\mathrm{B}$ ) sides of $\mathrm{P}(0)$ are indicted by negative and positive values of $\mathrm{X}$ in $\mathrm{P}(\mathrm{X})$, respectively. Figure 2 shows additional details of the experimental settings. Scale bars: $1 \mathrm{~cm}$ in all images. Spatiotemporal patterns of internode elongation in the eight shoots shown in the images $(\mathrm{B}, \mathrm{D}, \mathrm{F}, \mathrm{H}, \mathrm{J}, \mathrm{L}, \mathrm{N}$, and $\mathrm{P})$. At each $\mathrm{P}(\mathrm{X})$ position in each figure, the three bars from left to right indicate the relative growth at $\mathrm{P}(\mathrm{X})$ of the observed shoot at 4,8 , and 12 days after initial observation. In all images and figures, the positions with even numbers of $X$ and $P(0)$ are highlighted in light gray and dark gray, respectively. In the analysis, the distal ends were fixed as $\mathrm{P}(-2)$, whereas the proximal ends ranged from $\mathrm{P}(3)$ to $\mathrm{P}(6)$, depending on the length of the proximal internodes. 